\title{
DYNAMICAI HEATING OF THE UPPER ATMOSPHERE
}

\author{
C. 0. Hines, \\ Department of the Geophysical Sciences, \\ University of Chicago
}

Abstract. The energy that is deposited in the ionosphere, by internal atmospheric gravity waves propagating upward from below, is assessed on the basis of recent observational data. The implied heating rates are found to range from $10^{\circ} \mathrm{K} /$ day (near the $95-\mathrm{km}$ leve 1 ) to $100^{\circ} \mathrm{K} /$ day (near $140 \mathrm{~km}$ ), and they therefore compete with solar radiation as the primary source of heating in the ionospheric E region. The tidal input may be comparable, but its height of deposition is more difficult to assess. The residual wave energy that reaches the $F$ region carries a flux that may exceed $10^{-4}$ watt $/ \mathrm{m}^{2}$, so it may play a significant role in determining the heat budget of these higher levels. The waves should be accompanied by . reversible temperature fluctuations of $\pm 10^{\circ} \mathrm{K}$ and more, low in the $\mathrm{E}$ region, and they may therefore account for irregular temperature structure that has been reported.
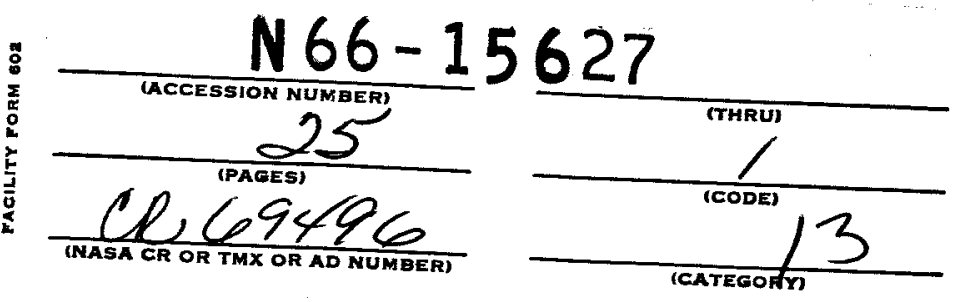

GPO PRICE

CFSTI PRICE(S)

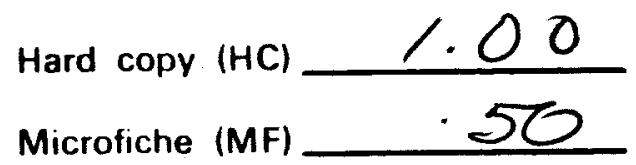

This paper has been prepared for submission to the Journal of Geophysical Research. It supersedes another of the same title, previously submitted to the Journal of Atmospheric Sciences, which will not proceed to publication. 
Introduction. The bulk of the atmosphere above the tropopause appears to receive its heat energy primarily through the absorption of optical radiation [Murgatroyd and Goody, 1958]. At very high altitudes, however, additional sources of heating are available and may become significant on occasion. One such source is provided by internal atmospheric gravity waves, which give evidence of propagating dynamical energy upward through the mesosphere into the ionospheric regions [Hines, 1960, 1963a, b].

The heating that may be caused by these waves is assessed in the present paper. It is found to be sufficient to modify temperatures in the E region, and may in fact account for the very rapid rise of temperature (with increasing height) that occurs there. Residual wave energy, propagating into the $\mathrm{F}$ region, contributes a small but sensible amount to the heat budget of the higher levels. Reversible temperature changes are also generated by the waves, of an amplitude that should be detectable and may already have been observed.

Earlier estimates. The present discussion follows upon another [Hines, 1963a] insofar as heating was concerned; that was limited fo heights near $95 \mathrm{~km}$. At those. levels, it was estimated, wave energy was being dissipated by molecular viscosity at a rate of about $5 \times 10^{-2}$ watt $/ \mathrm{kg}$, and a corresponding heating rate of a few degrees per day was inferred. (One watt $/ \mathrm{kg}$. will produce heating at a rate of $80^{\circ} \mathrm{K} / \mathrm{day}$, in an atmosphere of mean molecular mass 29 and a specific heat per molecule of 
$5 \times 10^{-23}$ joules $/{ }^{\circ} \mathrm{K}$. This estimate depended sensitively on the spectrum that was assumed for the distribution of wave energy, as between modes of different wavelengths, and that spectrum could be inferred only crudely.

At the same time, the then current estimate of turbulent dissipation near the $95-\mathrm{km}$ level, of $7 \times 10^{-3}$ watt $/ \mathrm{kg}$ [B]amont and de Jager, 1961; Greenhow, 1959], was noted as producing a heating of smaller but similar magnitude. An eddy viscosity about equal to ten times the molecular viscosity was also inferred, and would have implied a dissipation of wave energy at a rate of $5 \times 10^{-1}$ watt $/ \mathrm{kg}$. Though this energy would have to be dissipated by the turbulence in turn, and though it exceeded the estimate of turbulence dissipation by almost two orders of magnitude, the discrepancy was not considered to be serious in view of the gross uncertainties inherent in all the estimates and, indeed, in the very applicability of standard turbulence concepts.

Instead, the view was adopted that a reasonably consistent picture had been evolved, in which turtulence was maintained in being by the wave system, and in which a heating of a few degrees per day could be produced by the waves. This heating might result primarily by direct molecular dissipation, or it might proceed mainly via the intermediate process of turbulence. In either event, it was comparable to the average heating produced at the same levels by the absorption of solar radiation Mursatrovd and Goody, 1958], and must, of course, exceed the radiative input at night and in the winter polar regions (if a similar rate obtained). A secondary 
source of heat in the latter regions appears to be required, to offset the rapid radiative cooling that would otherwise result, and so to maintain the rather high temperatures that are indicated there [Murgatroyd and Goody, 1958]. Such a source could be provided by subsidence and related photochemical release [Kellogg, 1961; Maeda, 1963; Young and Epstein, 1962] or possibly hy scnustic heating [Maeda, 1964], but the role of internal gravity waves and associated turbulence appeared also to merit further examination.

New E-region estimates. It is now pospible to estimate the gravity-wave dissipation at middle latitudes by a totally independent means, and to extend the calculation upward in height. Most valuable, for this purpose is the analysis by Kochanski [1964] of the vertical variation of wave amplitude as revealed by numerous rocket-released vapor trails. This analysis is not wholly free from uncertainty, for the separation of waveindaced winds from the background was and will remain open to some ambiguity, but the results nevertheless constitute a tremendous advance and provide the best information of their type currently available.

Theory shows that, in an isothermal nondissipative atmosphere, free from background wind shear, the amplitude of the wave-induced horizontal wind speed $\left(U_{x}\right)$ should increase with altitude $(z)$ as the gas density $(p)$ decreases, the product $\rho U_{x}{ }^{2}$ remaining constant [ㅐines, 1960]. It was 
found by Kochanski [1964], however, that

$$
\rho U_{x}^{2} \propto \exp -\left(z / z_{0}\right)
$$

with

$$
z_{0}=7.6 \mathrm{~km}
$$

over the height range $70-140 \mathrm{~km} .\left(U_{x}\right.$ is represented by Kochanski as $W$, and the cited value of $z_{0}$ is derived from the exponential decrease of $\rho^{1 / 2} \times w^{\prime}$ described on p. 3659 of his paper.j

This observed decrease of wave energy with increase of height might be due in part to partial reflection, associated with the height variation of temperature or of background wind that occurs in practice; some such reflection could provide a reasonable explanation, in terms of a standing-wave component, for ionization stratifications that are reported to occur at certain preferred heights at lower levels (as reviewed, for example, by Ellyett and Watts [1959]).. On the other hand, viscous dissipation is known to be strong for the wavelengths observed [Hines, 1960], and much of the energy must be going into heat whether the remainder is reflected or not. This view is strengthened by the observed increase of minimum half-wavelength (with increasing height), as revealed by the vapor-trail analyses of Kochanski [1964, Fig. 8] and Zimmerman [1964], both of which give excellent agreement with the theoretical viscous cut-off [Hines, 1964]. The following calculation of energy dissipation neglects partial reflection, but its conclusions are unlikely to be in error by more than a factor of two on this account. 
Low in the E region, the bulk of the gravity-wave energy resides in modes whose vertical wavelength $\left(\lambda_{z}\right)$ is of the order $10 \mathrm{~km}$ [Kochanski, 1964]. This is consistent with the wavelength of $12 \mathrm{~km}$ inferred by Hines [1960] from various reports of meteor data, including that of Greenhow and Neufe1d [1959], and the wave period $(\tau)$ inferred from the latter report was about $1.2 \times 10^{4} \mathrm{sec}$. These waves would have a vertical component of group velocity $\left(\mathrm{V}_{\mathrm{z}}\right)$ about equai in magnitude to the vertical phase speed: $V_{z} \simeq \lambda_{z} / \tau \simeq 1 \mathrm{~m} / \mathrm{s}$. They oscillate nearly horizontally, and their mean energy density is given very nearly by $\rho_{\mathrm{x}}^{2} / 2$. Their vertical energy flux is given by

$$
F_{z}=\rho U_{x}^{2} v_{z} / 2
$$

and the vertical convergence of this flux, $-\mathrm{dF}_{z} / \mathrm{dz}$, yields the rate at which they are losing their energy. The vertical variation of $V_{z}$ that results from the vertical variation of temperature is negligible in comparison to the variation of $\mathrm{pU}_{\mathrm{x}}{ }^{2}$ given by (1) and (2), so the wave system appears to be losing energy at the rate $\rho U_{x}{ }^{2} V_{z} / 2 z_{0}$. When calculated for a unit mass of the atmosphere, the rate of energy loss becomes

$$
\varepsilon=U_{\mathrm{x}}^{2} \mathrm{v}_{\mathrm{z}} / 2 z_{0}
$$

Kochanski finds $U_{x}=40 \mathrm{~m} / \mathrm{s}$ as a mean amplitude at $95 \mathrm{~km}$, which combines with the values of $V_{z}$ and $z_{0}$ already given to yield an estimate of $\varepsilon \simeq 10^{-1}$ watt $/ \mathrm{kg}$ 
This estimate may be slightly high (because of partial reflections) or slightly low (because it makes no explicit provision for the energy borne by modes of smaller scale and shorter period, for most of which $V_{z}$ would be greater). It is, however, totally independent of the mechanism -molecular or turbulent -- that is assumed to be removing the wave energy, totally independent of coefficients that pertain to those mechanisms, and substantially independent of the detailed spectral distribution. Its agreement with the earlier estimates of wave dissipation, $5 \times 10^{-2}$ or $5 \times 10^{-1}$ watt $/ \mathrm{kg}$, is the more remarkable on this account. Even the discrepancy that persists, between it and the earlier estimate of turbulent dissipation, is sondwhat relieved by the higher values that are now being associated with turbulence (e.g., $1.5-3.5 \times 10^{-2}$ watt $/ \mathrm{kg}$ [Roper and E1ford, 1963; Noel, 1963], and even $7 \times 10^{-2}$ watt/kg [J. E. Blamont, private communication]).

Regardless of its route, the dissipated wave energy should appear ultimately as heat, and it implies a heating rate of the order $10^{\circ} \mathrm{K} / \mathrm{day}$. As previously argued, this heat input is comparable to the average radiative input, and its dominance over the latter at night and in the winter polar regions must be anticipated.

An even more striking conclusion can be reached for somewhat higher levels. Around $105-110 \mathrm{~km}$, Kochanski reports $\mathrm{U}_{\mathrm{x}} \simeq 55 \mathrm{~m} / \mathrm{s}$, which combines with earlier values to yield $\varepsilon \simeq 4 \times 10^{-1}$ watt $/ \mathrm{kg}$ or a heating of about $30^{\circ} \mathrm{K} /$ day.

$U_{x}$ decreases to $40 \mathrm{~m} / \mathrm{s}$ once again near the $140-\mathrm{km}$ level, but the waves that still remain observable as waves are confined to wavelengths $\lambda_{z} \gtrsim 30 \mathrm{~km}$. This is to be expected from viscous quenching criteria 
[Hines, 1960, 1964]. (Incidentally, the dissipation here can ro longer proceed via turbulence, for the turbulence terminates typically at heights $0: 100-110 \mathrm{~km}[\mathrm{e} .8 .$, Blamont and de Jager, 1961]. The increase of molecular kinematic viscosity more than makes up for the loss of eddy viscosity as a dissipative mechanism, however.) These quenching criteria further inäicare that the period to de associaced with the limiting $\lambda_{\mathrm{z}}$ (i.e., $30 \mathrm{~km}$ ) is no greater than $10^{3} \mathrm{sec}$, and the vertical phase speed is then close to $30 \mathrm{~m} / \mathrm{s} . \quad V_{z}$ will be somewhat less than this, but the energy density will Exceed $\rho \mathrm{u}_{\mathrm{x}}{ }^{2} / 2$ by a compensating factor. The implied dissipation rate exceeds $I$ watt $/ \mathrm{kg}$, and leads to a heating of the order $100^{\circ} \mathrm{K} / \mathrm{day}$. This conclusion must be modified by the fact that Kochanski reports the waves to be present at $140 \mathrm{~km}$ for only 50 percent of the time, but that conclusion in tur. must be qualified because his method of analysis is such as to minimize the role that is attributed to the larger-scale waves that are relevant here.

Doubtful though the exact value of the dynamical heating rate may be, there is no denying the implicstion that a very substantial heat input is available from internal gravity waves in the $E$ region. This heating, of course, exceeds the radiative input at night, and may very well exceed that input even when both are integrated over 24 hours. It may, in fact, be the primary cause for the very rapid rise of temperature with height that is found in the $\mathrm{E}$ region -- the relative rate of increase being there the maximum achieved in the whole of the atmosphere -- and, by limiting the flow of heat downward from the $F$ region, it may exert a strong influence on the temperature of the whole thermosphere. 
E-region heating. The direct input of wave energy to the $F$ region is worth evaluating, though the uncertainties increase once again. A density of about $3 \times 10^{-9} \mathrm{~kg} / \mathrm{m}^{3}$ obtains at $140 \mathrm{~km}$ [CIRA, 1961], and combines with other values already given to yield an energy flux of about $7 \times 10^{-5}$ watt $/ \mathrm{m}^{2}$ as the input to the overlying $F$ region, 50 percent of the time. This estimate could be low by as much as an order of magnitude, contributed by the 1 arger-scale waves that biend into the 'residual wind' in Kochanski's analysis. Such waves, if responsible for ionospheric traveling disturbances as has been argued [Hines, 1960], are in fact present most of the time [Heisler, 1963]. Their energy density could equal, and their $V_{z}^{\prime}$ 's tend to exceed, the values employed above. A mean energy flux exceeding $10^{-4}$ watt $/ \mathrm{m}^{2}$ is therefore a distinct possibility.

For purposes of comparison, it is usual to cite $10^{-3}$ watt $/ \mathrm{m}^{2}$ $\left(=1 \mathrm{erg} / \mathrm{cm}^{2} / \mathrm{sec}\right)$ as the rate at which the $\mathrm{F}$ region gives up its heat energy, via thermal conduction into the E region [Bates, 1951; Huxt and Van Zandt, 1961; Harris and Priester, 1962; Johnson, 1958]. Much of this energy is thought to be supplied by the extreme ultraviolet component of solar radiation, which carries a flux about three times as great [Hall et al, 1963; Hinteregger, 1961]. However, this flux is vertically incident only at the subsolar point, it is present at all only by day, and its conversion to heat is thought to be only 15-30 percent efficient [Chamberlain, 1961; Hanson and Johnson, 1961]. Its ability to meet the heating requirements of the $F$ region is doubtful, and no other solar radiations have been 
advocatec to make up the deficiency. Indeed, one analysis I Taris and 2ueser, 1962] suggests that a second heat source of quite different diurnal variation may be required to account for the observed density variations.

The calculation presented above gives some basis for looking to the dynamical ancrgy of intomal gravity waves as an auxiliany heat source for the $F$ region. It is certain, for one thing, that the entire energy flux that enters that region will degenerate to heat there. Moreover, because of variations in the (as yet unknown) sources of the waves, and. because of tical changes that will affect their passage through the E region, the flux will undoubtedly contain some diurnal modulation. Any speculation as to the phase or amplitude of such a modulation would be idle at the present time, however, and so will not be pursued here.

Nagnetic storms. At times of magnetic storms and active auroral displays, an aditional component of the gravity-wave spectrum may ie anticipated. This is because of the intensive heating that occurs in the auroral zones, whose spatial and temporal scales lie within the range that could efficiently coupie energy into the waves. It has been suggested by $T$. Gold Iprivate communication] that energy might be transported from the auroral zones to lower latitudes by means of the gravity waves, and thereby provide for the excessive heating that is revealed by satellites at such times [Jacchia, 1964]. 
This suggestion meets no particular difficulty with respect to travel times, for the energy could propagate to lower latitudes at speeds of almost 1000 $\mathrm{m} / \mathrm{s}$ in a ducted mode [cf. Press and Harkrider, 1962, Fig. 6] that is revealed, for example, by the gravity-wave component of the high-altitude nuclear blast waves reported by Obayashi [1962, note added in proof] and by a small but important class of ionospheric traveling disturbances found in the data of Tweten [1961] and Valverde [1958]. The magnitude of the effect is subject to uncertainty, however, and remains for further examination when more suitable data become available.

Reversible heating in the E region. The heating that has been treated to this point has been irreversible, the energy being lost from the wave system. However, until dissipation becomes severe in any given mode, that mode produces reversible, adiabatic heating, as one facet of its oscillation. The fractional temperature variations $(\delta \mathrm{T} / \mathrm{T})$ are related to $\mathrm{U}_{\mathrm{x}}$ by

$$
\delta \mathrm{T} / \mathrm{T}=(P-R) \mathrm{X}^{-1} \mathrm{U}_{\mathrm{X}}
$$

where $P, R$ and $X$ for a given mode are as defined by equations (15) - (17) or (23)- (25) of Hines [1960]. The full expressions are complicated, but reduce to the simpler form

$$
\delta T / T \simeq \pm i(\gamma-1)^{\frac{3}{2}} c^{-1} U_{x}
$$

when, as is the case for the dominant E-region waves, $\lambda_{z}$ does not exceed appreciably the local scale height of the atmosphere, and $\tau$ is substantially greater than $\tau_{g} \equiv 2 \pi \mathrm{Cg}^{-1}(\gamma-1)^{-\frac{1}{2}}$ (which is the Brunt-Vaissala period 
for an isothermal atmosphere). Here $\gamma$ is the ratio of specific heats, $C$ the speed of sound, and $g$ the acceleration due to gravity; the "i" indicates a phase quadrature between the time or place of maxima in $\delta \mathrm{T}$ and $U_{x}$, while the " \pm " uncertainty can be eliminated if the horizontal direction of phase propagation is known in relation to the direction of $U_{x}$. The temperature deviations that arise if $\tau$ tends toward $\tau_{g}$ exceed those indicated by $(6)$, provided the restriction on $\lambda_{z}$ is retained. The theory is based on an assumed isothermal atmosphere, but this idealization should not seriously restrict the validity of (5) and (6) as first approximations.

On the insertion of representative values into (6), it will be found that temperature fluctuations of $\pm 10^{\circ} \mathrm{K}$ can be expected low in the E region, and as much as $\pm 30^{\circ} \mathrm{K}$ at $110 \mathrm{~km}$, quite typically. Irregular temperature profiles just below the $100-\mathrm{km}$ level have been inferred by sound-ranging methods, with deviations often as large as $\pm 10^{\circ} \mathrm{K}$, and these deviations apparently exceed the probable error of measurement [ Smith et 3 ] 1964$]$. It is natural to suggest that they represent the reversible heating imposed dynamically by gravity waves, rather than quasi-static anomalies or purely spurious results. This suggestion is amenable to a detailed check (with respect to phase as well as amplitude) by refined programs of simultaneous temperature and wind sensing, and these programs could be extended to include density variations when measurement techniques improve slightly. Such analyses would go far towards consolidating or clarifying the picture of irreversible heating previously presented, in addition to their more direct benefits. 
Edal and prevailing-wind input. The atmospheric tides carry significant energy -nto the E region and distribute it through higher levels. They should not be ignored in any discussion of dynamical input, though their role is difficult to assess at present.

The semidiurnal tide at the base of the $E$ region exhibits a vertical variation of phase amounting to $3-7 \% / \mathrm{km}$ [Greenhow and Neufeld, 1961], which corresponds to a $\lambda_{z}$ of $50-120 \mathrm{~km}$. Higher in the $\mathrm{E}$ region, vapor trails reveal tidal $\lambda_{z}$ 's more of the order $30 \mathrm{~km}$ [Kochanski, 1964], and two sequences of trails [Rosenberg Edwards, 1964] suggests that this $\lambda_{z}$ should again be associated with the semidiurnal component. It would be convenient if these $\lambda_{z}{ }^{\prime} s$ were to correspond to the so-called '2, 2 ' mode of tidal oscillation that predominates at ground level and in 'resonance' theory, and whose associated 'effective height of the atmosphere' is $\mathrm{h}=7.9 \mathrm{~km}[\mathrm{e} . \mathrm{g}$. Wilkes, 1949]; but they don't, nor is there any good reason why they should [Hines, 1963a].

They imply, instead, modes with $\mathrm{h} \simeq 2.6 \mathrm{~km}$, when interpreted on the basis of conventional tidal theory, but their identification can be made no more precise than that. This is unfortunate, for the relation between observed winds and corresponding energy fluxes varies considerably from one mode to another, as an examination of the formulae (33), (34) and (39) of Wilkes [1949] will reveal. (The concept of a vertical group speed is no longer valid, because of the discrete nature of the spectrum of tidal modes.) Representative calculations may be carried out, nevertheless, and they suggest that the semidiurnal tidal flux upward into the E region is 
sbout $10^{-3}$ watt $/ \mathrm{m}^{2}$, while the energy density at $90 \mathrm{~km}$ is about $5 \times 10^{-3}$ joule/m $\mathrm{m}^{3}$ and so the effective vertical speed of energy flow is about $2 \mathrm{~m} / \mathrm{s}$. The figures are based on a speed of $20 \mathrm{~m} / \mathrm{s}$ for the semidiurral tidal wind at $90 \mathrm{~km}$ [Greenhow and Neufeld, 1961], on an $\mathrm{h} \simeq 4 \mathrm{~km}$, and on uncertain trigonometric factors which arise in the mode analysis and wich render the estimates of flux and speed suspect by a factor of four or so.

These values of flux, density and speed, are quite comparable to those of the gravity-wave system low in the $\mathrm{E}$ region. The tidal energy, if deposited there, should produce a heating of the sme coler / that already inferred for the gravity waves; if deposited at higher levels, the heating could be even greater.

This raises a further point of uncertainty with respect to the tides: the height of energy deposition. It seems quite clear that the energy is largely removed from the tidal wave itself below heights of $115 \mathrm{~km}$ [Fines, 1960]. Even when the wave penetrates to greater heights, as it does on occasion [e.g., Kochanski, 1964], it does not maintain anything like a constancy of $\rho_{\mathrm{x}}{ }^{2}$. The energy might be deposited in heat through the direct action of viscosity or through a cascading of energy in the gravity-wave spectrum, but hydromagnetic dissipation -- or, equivalently, tohe 'ohic Ioss' or 'joule heating' -- seems also to be a strong contender [Hines, 1963a]. In the course of such dissipation, however, electric fields are established and act to extend the whole tidal phenomenon upward to levels that the tidal wave itself is unable to reach (as revieirad by Fejer [1964] or Hines [1963a], for example). 
The extent to which the semidiurnal tide is in fact carried upward electrodynamically is by no means clear, conventional theory to the contrary. This is because conventional theory neglects any verical variation of tidal wind in the $\mathrm{E}$ region, whereas all recent information suggests that variation to be strong. Its effect would be to discharge, low in the $\mathbf{\Sigma}$ region, much of the electric field that would otherwise build up, and to produce there most of the ohmic losses of the system.

Iimited data exist to suggest that the diurnal tide exhibits little variation of phase with height [Greenhow and Hall, 1960], and, if confirmed, they may imply a local source for this tide rather than upward propagation through the mesosphere. Similarly, the prevailing wi-As of the $\mathrm{E}$ region would be generated primarily by local input, and should not be expected to reverse themselves within the E region. In any event, the diurnal tide [e.g. Kato, 1956] and the prevailing winds [van Sabben, 1962] are now receiving increasing attention as the likely sources of the quiet-day ionospheric current system, and it is their energy rather than that of the semidiurnal tide that would then control the electrodynamic system at higher altitudes.

Regardless of the driving agency, some estimate may be made of the joule heating that is associated with the observed magnetic variations. One calculation is provided by Cole [1962], and it implies a dissipation of $5 \times 10^{-5}$ watt $/ \mathrm{m}^{2}$, in a region centered on the $140 \mathrm{~km}$ level. This is certainly compatible with the semidiumal tidal flux cited above, while 
the appropriate figures for the diurnal and prevailing components are unknown. It is, moreover, as Cole points out, of possible significance to the heat budget of the higher levels. While it is less by an order of magnitude than the requisite $140-\mathrm{km}$ flux previously quoted, it is aiso uncertain by an order of magnitude. (The local dissipation rate to which this corresponds, at the $140-\mathrm{km}$ level, can be calculated only if an explicit assumption is made as to the magnitude of the associated electric fields. Representative calculations suggest the rate to be about $3 \times 10^{-1}$ watt $/ \mathrm{kg}$, which would produce a heating rate of about $25^{\circ} \mathrm{K} /$ day, but again with a substantial uncertainty.) of this final section, In summary $\mathcal{h}$ then, neither the propagation of tidal energy into the ionosphere, nor the electrodynamic transfer of tidal and prevailing wind energy within the ionosphere, should be neglected in any serious attempt at establishing the normal thermal budgets of the various upper atmospheric regions. But the specific regions in which these processes will be most significant, and the degree of their significance, cannot yet be established with useful precision.

Acknowledgments, The foregoing analysis was supported by the National Aeronautics and Space Administration, under grant NsG-467 Research. It originated in part, and has benefited, from discussions with J. E. Blamont, K. D. Cole, T. Gold, F. S. Johnson, W. W. Kellogg, G. A. M. King and W. Nordberg. 


\section{REFERENCES}

Bates, D. R., The temperature in the upper atmosphere, Proc. Phys. Soc., B, 64, 805, 1951 .

Blamont, J. E., and C. de Jager, Upper atmospheric turbulence near the $100 \mathrm{~km}$ level. Annales de Géophysique, 17, 134, 1961

Chamberlain, J. W., The energies in the spectra of the airglow and the aurora, Annales de Géophysique, 17,90, 1961.

CIRA, COSPAR International Reference Atmosphere 1961, Interscience Publishing Company, Inc., New York, 1961.

Cole, K. D., A source of energy for the ionosphere, Nature 194, 75, 1962.

Ellyett, C., and J. M. Watts, Stratification in the lower ionosphere, J. Res. Nat. Bureau Standards, 63D, 117, 1959.

Fejer, J. A., Atmospheric tides and associated magnetic effects, Rev. Geophys. 22, 275, 1964.

Greenhow, J. S., Eddy diffusion and its effect on meteor trails. J. Geophys. Res., 64, 2208, 1959.

Greenhow, J.S., and J. E. Hall, Diurnal variations of density and scale height in the upper atmosphere, J.Atmospheric Tetrestrial Phys. $18,203,1960$.

Greenhow, J. S., and E. L. Neufeld, Measurements of turbulence in the 80- to $100-\mathrm{km}$ region from the radio echo observations of meteors, J. Geophys, Res., $\underset{\sim 4}{2}, 2129,1959$. 
Gzeenhow, J. S., and E. L. Neufeld, Winds in the upper atmosphere, Quart. J, Roy. Met. Soc., 87, 472, 1961.

Hall, L. A., K. R. Damon, and H. E. Hinteregger, Solar extreme ultraviolet photon flux measurements in the upper atmosphere of August 1961, in Space Research III, edited by $w$. Priester, p. 745, North Hnlland Publishing Company: Amsterdam, 1963.

Hanson, W. B., and F. S. Johnson, Electron temperatures in the ionosphere, Memoires Soc. R. Liege, 5th series, $42390,1961$.

Harris, I., and W. Priester, Time-dependent structure of the upper atmosphere, J. Atmospheric Sci., 19, 286, 1962.

Heisler, L. H., Observation of movement of perturbations in the F-region, J. Atmospheric Terrestrial Phys., 25, 71, 1963.

Hines, C. O., Internal atmospheric gravity waves at ionospheric heights, Can. I. Phys., 38, 1441, 1960.

Hines, C. O., The upper atmosphere in motion, Quart. J. Roy. Met. Soc., $89,1,1963 a$.

Hines, C. O., Ionization irregularities in the E region, I, Atmospheric Terrestrial Phys., 25, 305, $1963 \mathrm{~b}$.

Hines, C. O., Minimum vertical scale sizes in the wind structure above 100 kilometers, J. Geophys. Res,, 69, 2847, 1964. 
Hinteregger, H. E., Preliminary data on solar extreme ultraviolet radiation in the upper atmosphere, J. Geophys. Res., 66, 2367, 1961.

Hunt, D. C., and T. E. Van Zandt, Photoionization heating in the F region of the atmosphere, J. Geophys, Res., 66, 1673, 1961.

Jacchia, L. C., Influence of solar activity on the earth's upper atmosphere, Planet. Space Sci., 12, 355, 1964.

Johnson, F. S., Temperatures in the high atmosphere, Annales de Géophysique, 14, 94, 1958.

Kato, S., Horizontal wind systems in the ionospheric $\mathrm{E}$ region deduced from the dynamo theory of the geomagnetic Sq variation, Part II, I. Geomag. Geoelectr., 8, 24, 1956.

Kellogg, W. W., Chemical heating above the polar mesopause in winter, J. Meteorology, 18, 373, 1961.

Kochanski, A., Atmospheric motions from sodium cloud drifts, J. Geophys. Res., 69, 3651, 1964.

Maeda, K., Auroral dissociation of molecular oxygen in the polar mesosphere, J. Geophys. Res., 68, 185, 1963.

Maeda, K., On the acoustic heating of the polar night mesosphere, J. Geophys. Res., 69, 1381, 1964. 
Murgatroyd, R. J., and R. M. Goody, Sources and sinks of radiative

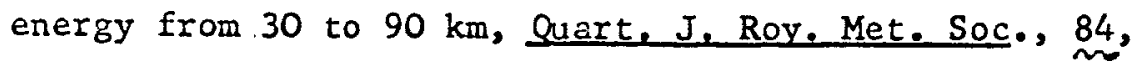
$225,1958$.

Noel, T. M., A measurement of turbulence power and small eddy scale near 105 kilometers, J. Geophys. Res., $\underset{\sim}{68}, 2862,1963$.

Obayashi, T., Widespread ionospheric disturbances due to nuclear explosions during October 1961, Rep. Ionosphere Space Res. Japan, 16, 334, 1962.

Press, F., and D. Harkridger, Propagation of acoustic-gravity waves in the atmosphere, J. Geophys. Res., 67, 3889, 1962.

Roper, R. G., and W. G. Elford, Seasonal variation of turbulence in the upper atmosphere, Nature, 197, 963, 1963.

Rosenbers, N. W., and H. D. Edwards, Observations of ionospheric wind patterns through the night, J. Geophys. Res., 69, 2819, 1964.

Smith, W., L. Katchen, P. Sacher, P. Swartz and J. Theon, Temperature, pressure, density and wind measurements with the rocket grenade experiment, 1960-1963, Report X-651-64-106. - NASA Goddard Space Flight Center, Greenbelt, Maryland, May 1964.

Tveten, I. H., Ionospheric motions observed with high-frequency backscatter sounders, J. Res. Nat. Bureau Standards, 65D, 115, 1961. 
Valverde, J. F., Motions of large-scale traveling disturbances determined from high-frequency backscatter and vertical incidence records, Sci, Rept, I, Radio Propagation Laboratory, Stanford Eiectronic Laboratories, Stanford, California, 1958.

vai Gabient, D., Ionosphoric current systems caused by non-periodic winds, I. Atmospheric Terrestrial Phvs., 24, 959, 1962.

Wilkes, M. V., Oscillations of the earth's atmosphere, University Press, Cambridge, 1949.

Young, C., and E. S. Epstein, Atomic oxygen in the polar winter mesosphere, J. Atmospheric Sci., 19, 434, 1962.

Zimerman, S. P., Small-scale wind structure above 100 kilometers, J. Geophys. Res., 69, 784, 1964. 